\title{
A REVIEW OF WOUND DRESSINGS - BIODEGRADABLE MATERIALS
}

\author{
Dr. Manjunatha $\mathbf{M}^{1}$ Vani Srinivasan ${ }^{2}$ \\ ${ }^{1}$ Department of Chemistry, CMR Institute of Technology, Bengaluru- 560037, India. \\ ${ }^{2}$ Department of Chemistry, Mount Carmel College, Bengaluru, Karnataka, India. \\ ${ }^{1}$ manjunatha.m@cmrit.ac.in ${ }^{2}$ vanis2410@gmail.com
}

\begin{abstract}
Wound dressing is one of the main external effectors during the healing process of wounds. Wound healing process recruits three different phases: inflammation, proliferation, and maturation. The main characteristics of the ideal wound dressing material is: control the moisture around wound, enable great transmission of gases, eliminate excess exudates, protect wound from infections and microorganisms, decrease surface necrosis of wound and have mechanical protection. There are currently many types of wound dressing biodegradable materials available in the market. Inspite of the wide selection, there is no universally superior dressing, which prompts healthcare providers to choose materials that are most suitable to each particular case. Modern dressings have evolved to encompass an expansive array of biodegradable films and foams, hydrofibers, hydrogels, hydrocolloids, and alginates. Recent studies have investigated new technologies such as electrospun biopolymer nanofibers to create sprayable dressings while also exploring new roles for traditional materials such as honey and other non-synthetic derivatives. This review provides an overview of various types of wound dressings, suggested uses and recent advancements in it. In addition, development of wound dressing biodegradable materials is the main objective of this paper which will provide general information on future wound dressing biodegradable materials.
\end{abstract}

Key words: Wound healing, biodegradable films, hydrofibers, hydrogels.

\section{INTRODUCTION}

An effective wound dressing should retain moisture in the wound, remove excess exudates, non-toxic, nonallergic, allow oxygen exchange, prevent microbes and be cost effective. Since there are different types of wound with various healing modes, this has led to research and development in introducing various types of wound dressings. The first wound dressing was introduced in Egyptian times when grease soaked guaze bandage was used to manage the wounds. Wound dressings before 1960's was passive with minimum healing process and when traditional dressings with gauze were used, they dried out soon but adhered to the wound causing bleeding and pain during its removal. Further, the exogenous bacterial entry could not be prevented by them although many layers of guaze were used. [1,2]

The awareness of active wound dressing and optimal environment in wound dressing came about after the groundbreaking research of Winter which led to the development of functional wound dressings from traditional ones. It was then, moist wound healing was adopted which had the advantage of soothing the nerve endings and hence removing the pain of the patient. But infection and slow healing was a real challenge, particularly in chronic diabetic ulcers and the true revolution in wound care came about in early 1980's when wound dressing with antibacterial activity for reducing bacterial infection was introduced. The antibacterial agents like carbolic acid, penicillin, mercuric chloride creams were used along with absorbent dressings. Silicone polymers replaced paraffin and trauma in the dressing changes were reduced.[3,4]

In the different dosage forms of wound care, patches result in skin irritation as they cause obstruction of sweat ducts and prevent loss of water vapour from skin surface. They cannot be applied on curved surfaces and also cause pain while peeling off. Creams and ointments instead of patches eliminate this disadvantage, but they are easily wiped off and need to be applied frequently for the healing to progress. This led to the innovations of synthetic as well as biological dressings.

Synthetic dressings can be films, foams and sprays, composite dressings, hydrocolloid dressings, granuflex, epiguard, biobrane, hydrogels etc. Biological dressings are from natural tissues usually containing collagen, elastin and lipids and are more superior than synthetic dressings. In film dressings, polymers play a very important role as they have the ability to form films with strong mechanical properties and can be doped with 
bioactive compounds. Both synthetic and natural polymers are utilized in making film dressings. They have advantages over liquid and semisolid drug delivery products due to their larger exterior area coverage, greater absorption of exudates, adhesive nature, low irritancy, low toxicity and prevention of loss of body fluids. Some of the natural polymers are alginate, chitosan, cellulose, starch, collagen and pectin. [5-9]

\section{DIFFERENT BIOMOLECULES IN WOUND DRESSING}

\section{BACTERIAL CELLULOSE BASED FILMS}

Bacterial cellulose is a natural polymer produced by microorganisms like acetobacter, agrobacterium, rhizobium etc and the carbon sources may be glucose, fructose, lactulose, corn stalk, nutshell etc.. The physico mechanical properties of $\mathrm{BC}$ make it an excellent wound healer but it lacks antibacterial properties. It is formed by continuous spinning of cellulose ribbons by bacteria leading to a 3D network structure stabilized by hydrogen bonds due to which it has high liquid loading capacity which is important for tissue regeneration. It also has high specific surface area compared to plant derived cellulose and hence its water absorption capacity is very high. Its biocompatibility, high tensile strength, good flexibility, high permeability to gas and liquid enhances its suitability for wound dressing. [10]

\section{BC/MMT FILMS}

Montmorillonite (MMT) is a widely used medicinal clay which is nontoxic without any side effects. It is used for cleaning and protection of skin, as well as it is active against E.coli, S.aureus. It also has blood clotting capability. Mazhar Ul-Islam et.al., in 2012 developed nanocomposite films of Bacterial Cellulose (BC) and Montmorillonite (MMT) which has high therapeutic value in wound healing and tissue regeneration. The composite does not allow MMT penetration in the blood which may lead to thrombosis. Hence they can be used for medical and drug delivery purposes. BC composites with pure MMT, Na-MMT, Ca-MMT and Cu-MMT were prepared and investigated against E.coli and S.aureus to analyse their uses as dressing material. $\mathrm{BC}-\mathrm{Cu}-$ MMT showed excellent antibacterial activity among them. It was also found that the antibacterial effects were greater in composites with higher MMT concentration. They have no side effects and hence can be utilized as an important tool in would healing. [11,12]

\section{Inorganic antibacterial agents- metal/metal oxide nano particles in $\mathrm{BC}$ based films}

BC combined with metal/metal oxide nano particles, nanosilicates and carbon nanoparticles has played important role in wound dressing and has been a research area till today. Janpetch et al., in 2016 synthesized $\mathrm{BC}-\mathrm{ZnO}$ composite and studied its antibacterial properties. The results has proved that BC is a very good polymeric template to carry $\mathrm{ZnO}$ nano particle. The composite was prepared by SPP method without the addition of any reducing agent which is the emerging green technology. The antibacterial activity depended on the concentration of $\mathrm{ZnO}$, its method of synthesis and the type of bacteria. Further investigations are necessary to study the safety of the composites.

$\mathrm{BC}$ oxidized with TEMPO or sodium periodate is treated with silver nitrate and Ag nano particles are synthesized on it by thermal reduction method. These composites are found to have high biocompatibility and antibacterial against E. coli and S. aureus which is a promising factor for wound dressing.

Au nanoparticles have excellent photothermal effect due to which local heat is generated on laser irradiation and the cell structure is destroyed. They bind to the ribosomes and prevent it from combining with t-RNA and destroy protein synthesis of bacterial cells. The metal oxide NPs like $\mathrm{ZnO}$ and $\mathrm{TiO}_{2} \mathrm{NPs}$ also have photocatalytic activity which can produce ROS under sunlight that kills bacteria.

\section{Organic antibacterial agents in cellulose based films}

The residues of nano particles after use can cause pollution and they may have long term potential toxicity problems. To overcome this drawback organic antibacterial natural polymers like chitosan, curcurmin, aminoacids etc and synthetic polymers like polyhexamethylene biguanidine blended with $\mathrm{BC}$ is used and is found to have very high biocompatibility and degradability.

Curcurmin is a natural polyphenol occurring in a herb called Curcuma longa. This compound has been under research over the years due to its non-toxic, antioxidant, antiinflammatory, anti-rheumatic, hypoglycemic and antibacterial activity. It is hydrophobic with very good wound healing property.

Gupta et al., 2019 encapsulated cucurmin in cyclodextrin and loaded into BC hydrogels and they found that its water solubility increased and it showed high blood compatibility, antistaphillococci and antioxidant property. 
Nanocellulose is derived from plants or bacteria which has good contraction property and it is an excellent absorber of exudates. Also it is known for its low toxicity and biodegradability. By accumulation of external matrix it accelerates wound recovery and hence it is used in deep wound and diabetic ulcer dressing.

Diabetic patients with foot ulcers, due to poor wound management show a high risk of amputation. High microbial load at the surface of the wound wrappings is a cause for the delayed wound healing. This is prevented by topical application of antimicrobial substance loaded film as wound dressing. Although silver ions are incorporated to serve this purpose, (Bitor et al 2014) it can lead to deposition of them in skin or eyes and also there is resistance of bacteria towards heavy metals. As a solution to this, cellulose nano crystal films with curcumin loaded in them was investigated by Woei Yenn Tong et al., in 2018 using diabetic rat models and it was noticed that the antimicrobial activity of the film was sustained even after 15 washes. The film showed inhibition on MRSA, B.coagulans, Streptococcus sp, E.coli, P.mirabilis and C.albicans.The bacterial growth reduction of $99.99 \%$ was observed from the skin sample of animal bodies. The regeneration of hair follicles and sebaceous glands improved in the case of curcurmin loaded film treatment and the results prove that they can be effectively used for wound healing in diabetic patients.

Zmejkoski et al., in 2018 developed BC -lignin dehydrogenate polymer composite (BC-DHP) hydrogel to treat chronic wounds and it could release DHP oligomer with antibacterial activity. [10-12]

\section{Amino acids in BC based films}

Among amino acids, lysine, arginine and tyrosine can promote cell proliferation and collagen deposition leading to wound healing.

Zywicka et al., in 2018 performed a coupling reaction with EDA based on Dilinoleic acid and Tyr forming a novel [EDA][DLA-Tyr] compound. BC incorporated with this compound showed long term antibacterial property againt S.aureus and S.epidermidis which is an excellent wound dressing. Quarternary ammonium salts possess both cationic part and hydrophobic segment which makes it an excellent penetrating agent and also it is antimicrobial agent with low toxicity and less skin irritation. Moreover, long chain unsaturated fatty acids are naturally known antibacterial agents. In the above experiment, dilinolinic acid and tyrosine which is capable of forming cation-pi interactions with Ethylene diamine was synthesized and incorporated into $\mathrm{BC}$ membrane. The increase in the length of the alkyl chain increased the antimicrobial property.[13]

He et al., in 2020 synthesised BC/PIL (polymer ionic liquids) composite composed of BC, and PILs formed by choline and different amino acids which showed antibacterial activity against gram positive, gram negative bacteria and fungi, hence a promising wound dressing. The preparation of the membrane involved only organic solvents and green synthesis. The free choline cation of PIL interact electrostatically with the cell membrane of the microorganism which is negatively charged which results in the probing of hydrophobic segments of PIL into the hydrophobic regions of the lipid membrane and leakage of cytoplasm resulting in cell death. The composite does not interact with the mammalian cell which is neutral. The molecular structure and concentration of PILs greatly affected its activity. PILs with strong hydrophobic benzyl and butyl groups showed high antimicrobial activity. The results showed that the anionic PILs exhibit sufficient antimicrobial activity.[14]

High capital investment, high operating costs and low yield are the major disadvantage of $\mathrm{BC}$ production and focus has to be made to overcome this and thus wound dressings have to be developed with low cost, simple process and no potential toxicity.

\section{ALGINATE BASED FILMS}

Alginate, which is a natural polysaccharide of seaweed is non-toxic and soluble in body fluids. It can interact with the exudates from the wound to form a hydrogel. It has the ability of swelling under moist conditions releasing encapsulated bioactive compounds.

Calcium alginate has hemostatic and antibacterial property which makes it an effective wound healer. Ciproflaxin loaded calcium alginate films were prepared by Asif Ahmed et al., in 2018 which showed ideal moisture absorption and maximum drug release within 90 minutes. It killed the bacteria in 24 hours. It was found to be very effective in treating diabetic foot ulcers with Staphylococcus aureus, Pseudomonas aeruginosa and Escherichia coli. CIP inhibits topoisomerase II and IV which are responsible for bacterial DNA replication. Tingting et al., in 2019 prepared ZnO/Sodium alginate bilayered hydrogel films. They incorporated zinc oxide nanoparticles into alginate hydrogel. These bilayered films showed high transparency, good hydrophilicity and swelling abilities. They have good antibacterial property and no significant cytotoxicity. Without hampering daily actions they can be applied to the wounds and it was demonstrated that they showed a higher wound healing ratio compared to the control. 
Jessica Miranda Rosa et al in 2018 prepared sodium alginate films in combination with essential oils/ oleoresin of six peppers that are commonly used in cooking. Essential oils are natural products from secondary metabolism of plants and present antioxidant, antifungal and antibacterial activity basically due to their hydrophobicity property. Antibacterial activity of the films were monitored over 30 days and they showed same inhibition zones for the entire period. Their antibacterial activity against E.coli, S. aureus and B.cereus is notable and this proves to be a new alternative for medicated wound dressings. [15-20]

\section{PVA, CHITOSAN AND STARCH BASED FILMS}

Poly Vinyl Alcohol is a non-carcinogenic, biocompatible, water- soluble synthetic polymer widely used in medicated films, membrane preparation, drug delivery, surgical materials, packaging etc.. It is biodegradable and of low cost. But it has some disadvantages like stiff film formation, in elastic film and limited hydrophilicity. When blended with oxidised maize starch which is a natural polysaccharide and which can be modified physically or chemically easily, they form excellent films. When antibacterial agents are added to PVA/Starch blend films they can serve as active packaging materials and in wound dressings. The polar secondary hydroxyl groups and geometric conformation of PVA ease its chemical modification. All the compounds that are able to react with the hydroxyl group can be used as probable cross-linking agents for PVA. PVA when blended with chitosan which is a natural polysaccharide with antimicrobial property and biocompatibility, the resulting biodegradable films are very effective packaging materials. Also, the binary and ternary blend films involving them has been studied and are found to be very promising wound healers.

Anuroop et al., developed medicated film using Chitosan and Hydroxy Propyl Methyl Cellulose (HPMC) as polymers with Silver sulphadiazine as the drug. In vivo studies on rats showed that the wounds healed within 8 days with medicated films whereas it took 14 days for healing with marketed creams. The film showed better biodegradability and bioadhesion. It serves as good protection to open wounds providing moist environment and repeated application of the cream is not required. It could be cut into any size to fit the wound size and it provides sustained release of the drug.[20]

PVA blend films with Coumarin derivatives and schiff bases is a promising future in wound dressings. Coumarin and its derivatives are known for their antioxidant, antimicrobial, antifungal, anti-parasitic and anticancer properties. Nowadays, hydroxy coumarin is used commonly as a precurser in organic synthesis owing to the presence of $\mathrm{OH}$ group in the $7^{\text {th }}$ position. When it is blended with PVA/chitosan or PVA/starch polymer the mechanical and antibacterial properties are enhanced and is suitable for antimicrobial food packaging and wound dressing.

Vinayak et al., in 2019 prepared 4-hydroxy coumarin doped PVC/chitosan (PC4HC) films by solution casting technique. The hydrophilic nature of the PC films changed to hydrophobic after the addition of 4-HC. The glass transition temperature shifted to higher values on adding $4 \mathrm{HC}$ and it became hydrophobic as $-\mathrm{OH}$ and $-\mathrm{NH}_{2}$ groups on PVA and chitosan are involved in hydrogen bonding. The films showed high tensile strength which is attributed to the intermolecular hydrogen bonding between $-\mathrm{NH}_{3}{ }^{+}$of the $\mathrm{CS}$ backbone and $-\mathrm{OH}$ of the $4 \mathrm{HC}$. These results show that it has potential application in wound dressing.[21]

Vishram D. Hiremani et al., in 2020 prepared 7-hydroxy-4-methyl coumarin doped PVA/Starch blend films. Starch/PVA blend plastics are well known biodegradable plastics used in packaging and agricultural applications. Incorporating 7H4MC with PVA/Starch blend promoted the mechanical properties of the film due to intermolecular and intramolecular hydrogen bonds between the polar hydroxyl and carbonyl groups in their chemical structure. It exhibited good antioxidant properties and hence has a promising future in active food packaging. Its antibacterial properties need to be explored and further research is ongoing for its applications in wound dressing.[22]
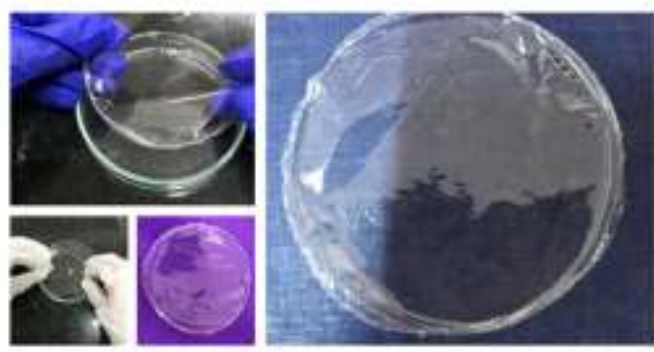

Awais Hassan et al., in 2017 prepared hydrogel membranes by cross linking PVA with starch using glutaraldehyde. Turmeric was added to it as antibacterial agent. The hydrogen bond interaction in it improved its mechanical properties and hence can be used in wound dressing.

Schiff bases are known for their high antibacterial properties due to the presence of azomethine group in SB contributing to the enhancement of antibacterial activity of the starting compounds from which it is prepared. A curious thought of blending it with PVA/Starch films could result in medicated films of high antibacterial activity which is required in chronic and diabetic wound dressings. Research is going on in this field and it has wide scope in the upcoming years. [23,24]

Vitamins, propolis and plant extracts in PVA based films 
Vitamin C is an antioxidant actively involved in collagen formation and also in the immune system modulation. The pleasant smelling Propolis also called as bee glue, is a resinous substance collected by bees from different plants. It is a natural antimicrobial, antioxidant, anticancer, antiulcer and anti-inflammatory agent improving wound healing by epithelialization.

In diabetic patients, wound healing is a real challenge as it takes longer time for the healing process and it is painful. A new therapeutic eco-friendly approach to cure such wounds was undertaken by Guilherme T. Vossa et al.,2018 who prepared cellulose based film loaded with vitamin-C, investigated the antibacterial and wound healing properties of them in diabetic an non diabetic mice. Cel-PVA/VitC and Cel-PVA/VitC/Prop films were prepared and vitamin $\mathrm{C}$ was released from these films in a controlled manner. In vivo experiments suggested a synergistic effect of vitamin $\mathrm{C}$ and propolis driving the wound healing phenomenon. The wound healing was enhanced without altering the pathological condition of the diabetic mice.

Aloe vera is a colourless gel obtained from parenchyma leaf and it is used to treat burns besides from being used as moisturizer. It alleviates pain and heals the skin. Vitamin E, a natural antioxidant has in it tocopherols and tocotrienols which have direct effect on tissue re pair and regeneration.

Gabriela Garrastazu Pereira et al., 2013, prepared bioadhesive PVA/Sodium Alginate films loaded with Aloe vera and vitamin $\mathrm{E}$ acetate, tested it on healthy volunteers. By tape stripping technique it was found that this film facilitates the more deeper accumulation of vitamin $\mathrm{E}$ acetate in stratum corneum and showed controlled release of the vitamin for more than 10 hours. This is found to be an innovative approach for the treatment of burns.[25,26]

Wound dressing containing natural compounds is gaining prominence nowadays and recently Nubya Nascimento Costa et al., 2020, prepared PVA/starch/PAA films containing pomegranate peel extract that can serve as temporary bioactive wound dressing material. The film showed antimicrobial activity against $\mathrm{S}$. epidermidis and $\mathrm{S}$. aureus with inhibition zones $>18 \mathrm{~mm}$. The addition of the extract contributed to the stability and integrity of the film by promoting interactions between the polymer chains. In vitro wound healing tests showed that the films containing $1.25 \%$ of the extract were efficient enough in closing the scratches completely in just 48 hours.[27]

Honey, Propolis and Royal jelly from honey bees are very good wound healers. Research in this field with further investigations of incorporating them in the wound dressing is essential, envisaging developments in this field. [28]

\section{WOUND DRESSINGS WITH STIMULI-RESPONSE}

In traditional wound dressings excessive release of antibiotics by passive diffusion can lead to drug resistance which can hinder the healing process. Discovery of various stimuli responsive wound dressings came as a solution to this problem.

Silver sulphadiazine has a wide range of antibacterial and antifungal properties which is used commonly in burn management. Its mechanism of action is by release of $\mathrm{Ag}+$ ions and when it is ionised too much it may lead to cytotoxicity.

Shao et al., in 2016 clubbed Silver sulphadiazine with BC forming a matrix that has pH controlled release behaviour and good antibacterial activity against S. aureus and C.albicans. AgSD in aqueous solution gives $\mathrm{Ag}^{+}$ and $\mathrm{SD}^{-}$. The release of $\mathrm{Ag}$ ions and SD ions was found to be faster at lower $\mathrm{pH}$ levels. But compared to silver ions SD ions were released at a much faster rate the reason being the interaction of silver ions with the hydroxyl group of BC. SD ions do not interact with BC matrix. Thus the release of high concentration of silver ions and hence its toxicity could be avoided. Also, the release behaviour of free AgSD and AgSD loaded into BC were compared and the latter showed slow release activity. The $\mathrm{pH}$ sensitive controlled release of silver ions and SD ions from the BC-AgSD composite is the advantage which could satisfy our expectations of controlled release of drug in wound therapy. [29]

Anandhakumar et al., in 2016, prepared polyelectrolyte multi-layered (PEM) films with weak polyelectrolytes for transdermal multi-drug delivery. The film was prepared by assembling layer by layer poly(allylamine hydrochloride)(PAH) and poly(methacrylic acid) (PMA) and it was loaded with bovine serum albumin (BSA) ciprofloxacin hydrochloride $(\mathrm{CH})$. The morphology of the film changed from pore free to porous film due to charge imbalances in the film in response to changes in $\mathrm{pH}$ and ionic strength favouring the sustained release of BSA and $\mathrm{CH}$ for about 8 hours. The loading was found to be $40 \%$ higher when compared to the films made of strong polyelectrolytes, i.e., PAH/dextran sulfate films whose surface morphology was not sensitive to changes in $\mathrm{pH}$ and ionic strength. In vitro cell culture experiments showed that these PEM films are highly biocompatible. They have the potential to be effective as antimicrobial and anti-inflammatory coatings as well as drug releasing coatings. [30]

Qian Pang et al., in 2017 developed LHP loaded PVA/SA wound dressing fabricated through freezing-thawing cycle process. The poly prodrug LHP was obtained by conjugating levofloxacin with PEG diamine using a 
ortho-nitrobenzyl linker, 4-(4-(1-Hydroxyethyl)-2-methoxy-5-nitrophenoxy) butyric acid (HMNB). The photo cleavable LHP was loaded into PVA/SA wound dressing from which the antibiotic LF (Levofoxacin) was released at $365 \mathrm{~nm}$ gradually on constant exposure to UV radiation. On irradiation of UV light, the ester bond in LHP broke and resulted in the release of LF. The release of LF increased proportionately and after about 30 minutes the release profile became flat. This was attributed to cleavage of all the esters in an half an hour duration. This stops the excess antibiotic release which is very important to avoid drug resistance in the patient. The dressing also showed high swelling capacity and very good cytocompatibility compared to the PVA/SA wound dressing as LHP decreases the cross linking density of PVA and alginate chains. [31]

\section{COLLAGEN BASED FILMS}

Collagen is a natural biopolymer in mammal's body which is the structural protein present in the extracellular space in the various connectivity tissues. Collagen based dressings are more common in places of high humidity as closed dressings may lead to septic of the wounds and topical creams become may become dry. CLRI, government of India in Chennai manufactures collagen membrane dressings. It is drawn out from bovine skin and Achilles tendons and it is redesigned. The new tissue ingrowth into the fibrous form of collagen resulting in its adhesive property is the benefit of using collagen in burn coverings.

Mathangi et al., in 2013 studied the collagen film doped with silver sulphadiazine loaded Alginate globules for chronic infected burns wound treatment and it was found that SSD delivered in a controlled manner.

Films made of collagen by casting collagen in methacrylate surfaces, cross linked with uv irradiation and embedded by nylon mesh, showed rapid epithelialization and thick fibrosis ingrowth in subdermal layers. Collagen forms the substrate needed for adhesion of cells and their migration. [32-38]

\section{CONCLUSION}

This review provides insight into the research and developments in wound dressing, advantages and disadvantages of various types of biomolecules in wound dressing and their unique properties. Developing wound dressings of low cost, with excellent physical and chemical properties, low toxicity, safe and green with fast wound recovery potential and overcoming drug resistance is the main objective of this paper. Incorporating Schiff bases would rather improve the properties of the medicated films and is a new approach available for the present and future research in the development of wound dressings.

\section{REFERENCES}

1. Douglas Queen, Heather Orsted, Hiromi Sanada, Geoff Sussman, A dressing history - Review, International Wound Journal. $2004 ; 1(1)$

2. Sipos P, Gyory H, Hagymasi K, Ondrejka P, Blazovics A, Special wound healing methods used in ancient Egypt and the mythological background., World J Surg. 2004;28(2):211-6

3. Winter GD, Formation of the scab and the rate of epithelialization of superficial wounds in the skin of the young domestic pig, Nature 1962;193:293 \pm 4 .

4. Alvarez O, Moist environment for healing: matching the dressing to the wound., Ostomy Wound Manage. 1988;21:64-83.

5. Park GB, Burn wound coverings a review. Biometer Med Devices Artif Organs. 1978;6:1.

6. Davies JWL, Synthetic materials for covering burn wounds progress towards perfection parts I \&

II. Burns 1983;10:94 and 104. 
7. Wong P, Physical evaluation of hydrogel as a burn wound dressing. MSc, Thesis. University of Strathclyde, 1980 .

8. Rehman MM, In vitro assessment of polymeric burn wound coverings. MSc, Thesis. University of Strathclyde, 1982.

9. Quinn KJ, Courtney JM, Evans JH, et al., Principles of burn dressings. Biomaterials. 1985;6:369.

10. Lu Zheng, Shanshan Li, Jiwen Luo and Xiaoying Wang, Latest Advances on Bacterial CelluloseBased Antibacterial Materials as Wound Dressings, Biomaterials, Frontiers in Bioengineering and Biotechnology. 2020; 8:593768.

11. Sibusiso Alven, Xhamla Nqoro and Blessing Atim Aderibigbe, Polymer-Based Materials Loaded with Curcumin for Wound Healing Applications, Polymers 2020;12:2286

12. Sadeghianmaryan, A.; Yazdanpanah, Z.; Soltani, Y.A.; Sardroud, H.A.; Nasirtabrizi, M.H.; Chen, X. Curcumin-loaded electro- spun polycaprolactone/montmorillonite nanocomposite: Wound dressing application with anti-bacterial and low cell toxicity properties., J. Biomater. Sci. Polym. Ed. 2020;31:169-187

13. Anna Zywicka, Karol Fijałkowski,* Adam F. Junka, Jakub Grzesiak, and Miroslawa El Fray, $\begin{array}{llll}\text { Modification } & \text { of } & \text { Cellulose } & \text { with }\end{array}$ QuaternaryAmmoniumCompoundsBasedonFattyAcidsandAminoAcidsandtheEffecton Antimicrobial Activity, ACS publications.

14. Xiaoling He,* Yuqing Yang, Haode Song, Shuai Wang, He Zhao, and Dongsheng Wei* Polyanionic Composite Membranes Based on Bacterial Cellulose and Amino Acid for Antimicrobial Application, ACS Appl. Mater. Interfaces 2020;12:14784-14796

15. Chem PL, Baum CL, Arpey CJ, et al. Biologic dressings: Current applications and limitations in dermatologic surgery. Dermatol Surg. 2009;35:891-906.

16. Bale S, Baker N, Crook H, Rayman A, Rayman G, Harding KG. Exploring the use of an alginate dressing for diabetic foot ulcers., J Wound Care 2001;10(3):81—4.

17. M. D. M. Dantas, D. R. R. Cavalcante, F. E. N. Arau'jo et al., "Improvement of dermal burn healing by combining sodium alginate/chitosan- based films and low level laser therapy," Journal of Photochemistry and Photobiology B, 2011;105:1:51-59. 
18. Ishihara M, Nakanishi K, Ono K, Sato M, Kikuchi M, Saito Y, Yura H, Matsui T, Hattori H, Venoyama M, Kurita A. Photocrosslinkable chitosan as a dressing for wound occlusion and accelerator in healing process.,Biomaterials, 2002;23(3):833-40.

19. L.J. Borda, F.E. Macquhae, R.S. Kirsner, Wound dressings: a comprehensive review., Curr. Dermatol. Rep.2016;5(4):287-297, https://doi.org/10.1007/s13671-016- 0162-5.

20. U. P. Anuroop, Sruthy S. Nair and A. J. Chacko, Development and evaluation of medicated biodegradable film for wounds and burns IJPSR. 2021;12:6.

21. Vinayak N. Vanjeri , Naganagouda Goudar, Deepak Kasai , Saraswati P. Masti , Ravindra B. Chougale, Thermal and tensile properties study of 4-Hydroxycoumarin doped Polyvinyl alcohol/Chitosan blend films, Chemical Data Collections.2019;23:100257:1-12

22. Vishram D. Hiremani, Sarala Sataraddi, Praveen K. Bayannavar,Tilak Gast, Saraswati P. Masti, Ravindra R. Kamble,Ravindra B. Chougale, Mechanical, optical and antioxidant properties of 7Hydroxy-4-methyl coumarin doped polyvinyl alcohol/oxidized maize starch blend films, SN Applied Sciences.2020; 2:1877:1-18

23. Tudorachi N, Cascaval N, Rusu M, Pruteanu M (2000) Testing of polyvinyl alcohol and starch mixtures as biodegradable polymeric materials. Polym Test.2000; 19:785-799.

24. Jayasekara R, Harding I, Bowater I, Christie GBY, Lonergan GT Preparation, surface modification and characterisation of solution cast starch PVA blended films., Polym Test.2004; 23:17-27

25. T. Reynolds and A. C. Dweck, “Aloe vera leaf gel: a review update,” Journal of Ethnopharmacology, 1999; 68:1-3.

26. S. Choi and M.-H. Chung, "A review on the relationship between Aloe vera components and their biologic effects," Seminars in Integrative Medicine.2003;1: 53-62.

27. P. Panichayupakaranan, S. Tewrakul, S. Yuenyongsawad, Antibacterial anti-in- flammatory and antiallergic activities of standardized pomegranate rind extract, Food Chem.2010;123(2) :400403. https://doi.org/10.1016/j.foodchem.2010. 04.054.

28. Visweswara Rao Pasupuleti, Lakhsmi Sammugam, Nagesvari Ramesh, Siew Hua Gan. Honey, Propolis, and Royal Jelly: A Comprehensive Review of Their Biological Actions and Health Benefits., Oxid Med Cell Longev. 2017;1259510. 
29. Wei Shao, Hui Liu , Jimin Wu, Shuxia Wang, Xiufeng Liu , Min Huang, Peng Xu, Preparation, antibacterial activity and pH-responsive release behavior of silver sulfadiazine loaded bacterial cellulose for wound dressing applications, Journal of the Taiwan Institute of Chemical Engineers 63 (2016) 404-410

30. S. Anandhakumar, P. Gokul, A.M. Raichur, Stimuli-responsive weak polyelectrolyte multilayer films: A thin film platform for self-triggered multi-drug delivery, Materials Science and Engineering C 58 (2016) 622-628

31. Qian Pang, Xiaowen Zheng, Yu Luo, Lie Ma and Changyou Gao, A photo-cleavable polyprodrugloaded wound dressing with UV-responsive antibacterial property, Journal of Material Chemistry, 2017. DOI: $10.1039 / \mathrm{c} 7 \mathrm{tb} 01696 \mathrm{~d}$

32. Balasubramani M,Kumar TR, Babu M. Skin substitutes: A review. Burns, 2001;27:534-44.

33. Singh O, Gupta SS, Soni M, Moses S, Shukla S, Mathur RK, Collagen dressing versus conventional dressings in burn and chronic wounds: A retrospective study. J Cutan Aesthet Surg. 2011;4:12-16.

34. Brett D. A review of collagen and collagen-based wound dressings. Wounds. 2008;20:12.

35. Shoshan S, Gross J. Biosynthesis and metabolism of collagen and its role in tissue repair processes. Israel J Med Sci. 1974;10:537-61.

36. Pruitt BA, Levine NS Jr. Characteristics and uses of biologic dressings and skin substitutes. Arch Surg. 1984;119:312-22.

37. Chem PL, Baum CL, Arpey CJ, et al. Biologic dressings: Current applications and limitations in dermatologic surgery. Dermatol Surg. 2009;35:891-906.

38. Bao L, Yang W, Mao X, Mou S, Tang S, Agar/collagen membrane as skin dressing for wounds. Biomed Mater. 2008;3:4. 